\title{
Heavy element Nucleosynthesis in the MHD Jet Explosions of Core-Collapse Supernovae
}

\author{
Nobuya Nishimura* \\ Department of Physics, Kyushu University, 1-2-4 Ropponmatsu, Fukuoka 810-8560, Japan \\ E-mail: nobuya@gemini.rc.kyushu-u.ac.jp
}

\section{Masa-aki Hashimoto}

Department of Physics, Kyushu University, 1-2-4 Ropponmatsu, Fukuoka 810-8560, Japan

\section{Shin-ichiro Fujimoto}

Department of Electronic Control, Kumamoto National College of Technology, Kumamoto 861-1102, Japan

\section{Kei Kotake}

National Astronomical Observatory of Japan, 2-21-1 Osawa, Mitaka, Tokyo 181-8588, Japan

\section{Shoichi Yamada}

Science and Engineering, Waseda University, 3-4-1 Okubo, Shinjuku, Tokyo 169-8555, Japan

\begin{abstract}
Massive stars whose mass exceed 10 times the solar mass evolve until the Fe-core is formed. Since the nuclear reactions in the Fe-core do not proceed further, the core begins to collapse, which leads to the catastrophic explosion. The nucleosynthesis during the evolutions and subsequent explosions is responsible for the heavy element enrichment in our galaxy. We calculate heavy element nucleosynthesis during the magneto-hydrodynamical (MHD) explosion of supernova of a massive star. We consistently calculate all stages of the collapse of the Fe-core, the core bounce, and the shock wave propagation. When the shock wave propagate in the oxygen-neon layers, the $p$-process nucleosynthesis should occur. Until now, the p-process has been studied with use of spherical explosion models where significant deficiencies in some light $p$-elements compared to solar ones have been found. We present nonspherical effects on the production of p-elements using adiabatic MHD explosion model.
\end{abstract}

International Symposium on Nuclear Astrophysics - Nuclei in the Cosmos - IX

25-30 June 2006

CERN

\footnotetext{
* Speaker.
} 


\section{Introduction}

Massive stars of $\mathrm{M}>10 M_{\odot}$ evolve until the Fe-core is formed. Since the nuclear reactions in the Fe-core do not proceed further but the photodisintegrations of nuclei occur, the core begins to collapse, which leads to the catastrophic explosion. Heavy 35 stable nuclei $(Z \geq 34)$ that are not produced by the successive neutron captures are classified as $p$-elements. The nucleosynthesis process to produce $p$-elements is the $p$-process which occurs during the supernova explosion. The most important astronomical site of the p-process is the explosion of massive stars, that is, corecollapse supernova [1].

In this paper, we investigate the $p$-process nucleosynthesis during the core collapse supernova explosion which we consistently calculate all stages of the collapse of the Fe-core, the core bounce, and the shock wave propagation. We perform the MHD simulation with the assumption of the adiabatic explosion [2], where the stellar rotation and magnetic field are included in a presupernova model of $13 M_{\odot}$ star.

\section{Presupernova and explosion models}

The presupernova model is calculated from the evolution of a He-core of $3.3 M_{\odot}$ which corresponds to $13 M_{\odot}$ in the main-sequence stage [3]. The mass of the Fe-core is $1.18 M_{\odot}$ that is the smallest Fe-core in massive stars obtained from the stellar evolution calculation with the limitation of spherical symmetry. The edge of the Fe-core which has a steep density gradient is at $R=8.50 \times 10^{7} \mathrm{~cm}$ from the center. The mass of the Si-rich layer is $0.33 M_{\odot}$ and the layer extends to $5.47 \times 10^{8} \mathrm{~cm}$ above the Fe-core. The O/Ne-rich layer extends to $0.31 M_{\odot}$ and $2.95 \times 10^{9} \mathrm{~cm}$ above the Si-layer. Since the central density exceeds $10^{10} \mathrm{~g} \mathrm{~cm}^{-3}\left(\rho=2.79 \times 10^{10} \mathrm{~g} \mathrm{~cm}^{-3}\right)$ and temperature $T_{9}=9.04$ in units of $10^{9} \mathrm{~K}$, the Fe-core just begins to collapse.

Initial models (precollapse models) are constructed by using the density and temperature distributions of the original presupernova model that contains $\mathrm{Fe}, \mathrm{Si}$ and $\mathrm{O} / \mathrm{Ne}$ layers. The $p$-process occurs using the seed of $s$-elements produced during the core helium burning of massive stars. We use the available data of heavy elements as the seed nuclei obtained from the previous $s$-process calculation for the star of $M=10 M_{\odot}$. We adopt cylindrical properties of the angular velocity $\Omega$ and the toroidal component of the magnetic field $B_{\phi}$, as follows [4]:

$$
\begin{aligned}
& \Omega(X, Z)=\Omega_{0} \frac{X_{0}^{2}}{X^{2}+X_{0}^{2}} \frac{Z_{0}^{4}}{Z^{4}+Z_{0}^{4}}, \\
& B_{\phi}(X, Z)=B_{0} \frac{X_{0}^{2}}{X^{2}+X_{0}^{2}} \frac{Z_{0}^{4}}{Z^{4}+Z_{0}^{4}},
\end{aligned}
$$

where $X$ and $Z$ are the distances from the rotational axis and the equatorial plane with $X_{0}$ and $Z_{0}$ being model parameters. Both $\Omega_{0}$ and $B_{0}$ are the initial values at $X=0$ and $Z=0$. Since the value of $T /|W|$ is higher compared to that used in [5] by a few percents, we regard the present case of $T /|W|=0.5 \%$ as rather rapid rotating stars with the moderate magnetic field.

In the computation, spherical coordinates $(r, \theta)$ are adopted. The computational region is set to be $0 \leq r \leq 4,000 \mathrm{~km}$ and $0 \leq \theta \leq \pi / 2$, where the included mass in the precollapse models 

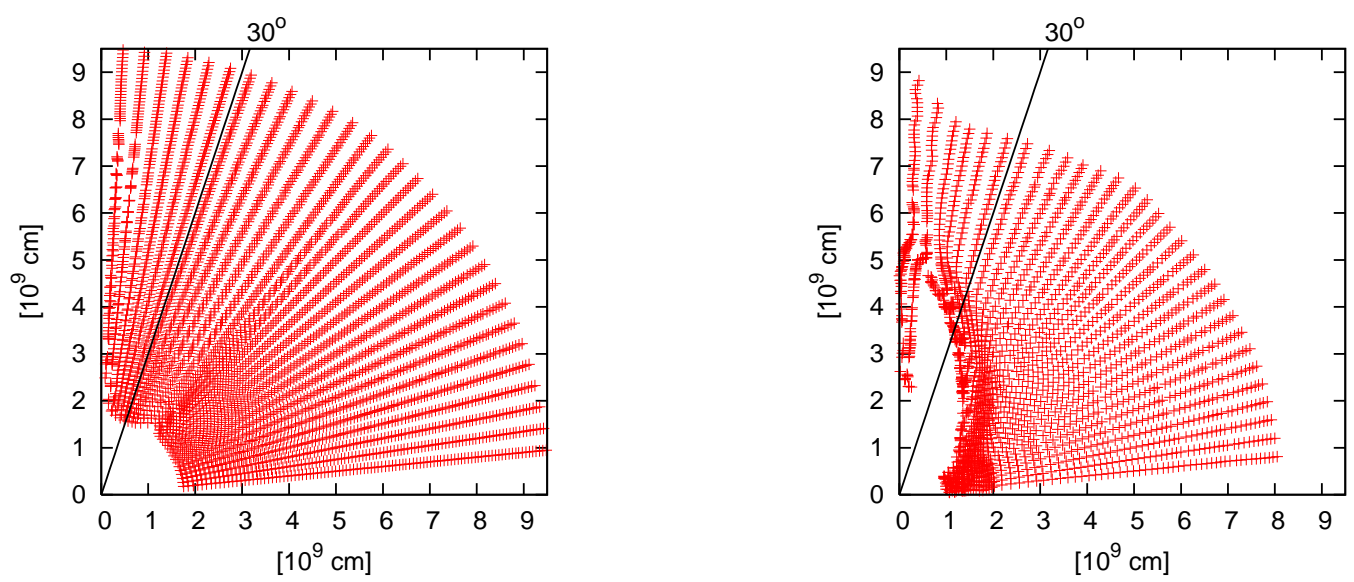

Figure 1: Location of the tracer particles. Each point shows the particle. Left figure shows the configuration when the shock wave reach Si-layer $(0.5 \mathrm{sec})$ and right figure shows shock wave propagate $\mathrm{O} / \mathrm{Ne}$-ayers $(2.0$ sec).

amounts to $1.42 M_{\odot}$. Then we add $\mathrm{O} / \mathrm{Ne}$ layer $(\leq 20,000 \mathrm{~km})$ after the shock wave reaches $\mathrm{Si}$ layer. The first quadrant of the meridian section is covered with $400(r) \times 30(\theta)$ mesh points. To get information of mass elements for $p$-process calculations, tracer particles are placed within the region of $\mathrm{O} / \mathrm{Ne}$-layers between $0.8 M_{\odot}(r=410 \mathrm{~km})$ and $1.3 M_{\odot}(r=2200 \mathrm{~km})$. More details for the numerical method are written in [2], where the $r$-process occurs in the inner region.

\section{Jet propagation to the outer layer: $p$-process nucleosynthesis}

It has been shown that the $p$-process occurs by the explosion in the layers where the peak temperature becomes $2-3 \times 10^{9} \mathrm{~K}[1]$. When the shock wave passes through the oxygen rich layer, the temperatures behind the shock reach these values. The final products depend on the peak temperature. MHD jet models are affected by the stellar model, and the directions of the jet determine the peak temperatures. Thus, the $p$-process products must be different from those produced in the spherical explosions depending on the direction.

Figure 1 shows the motion of tracer particles. The particles that are located in the oxygen layer extends first with the propagation of the shock wave. We obtain the time evolution of the density and temperature for each particle. Then, we calculate the nucleosynthesis from each particle. We use nuclear reaction network that includes 1989 nuclides for the $p$-process calculations [6.

From nuclear reaction network calculations, we can see the time evolution of the nucleosynthesis. Figure 2 shows the abundance flow for the particles located initially in the direction of 15 degree form the rotational axis. Figure 3 shows the overproduction factor relative to the solar abundances for two directions.

\section{Conclusion}

We investigate the $p$-process nucleosynthesis during the MHD explosions of supernovae in a massive star of $13 M_{\odot}$ without neutrino effect. During the MHD shock wave propagation in the oxygen-neon layers, the $p$-process occurs inside the MHD jets. Until now, the $p$-process has been 

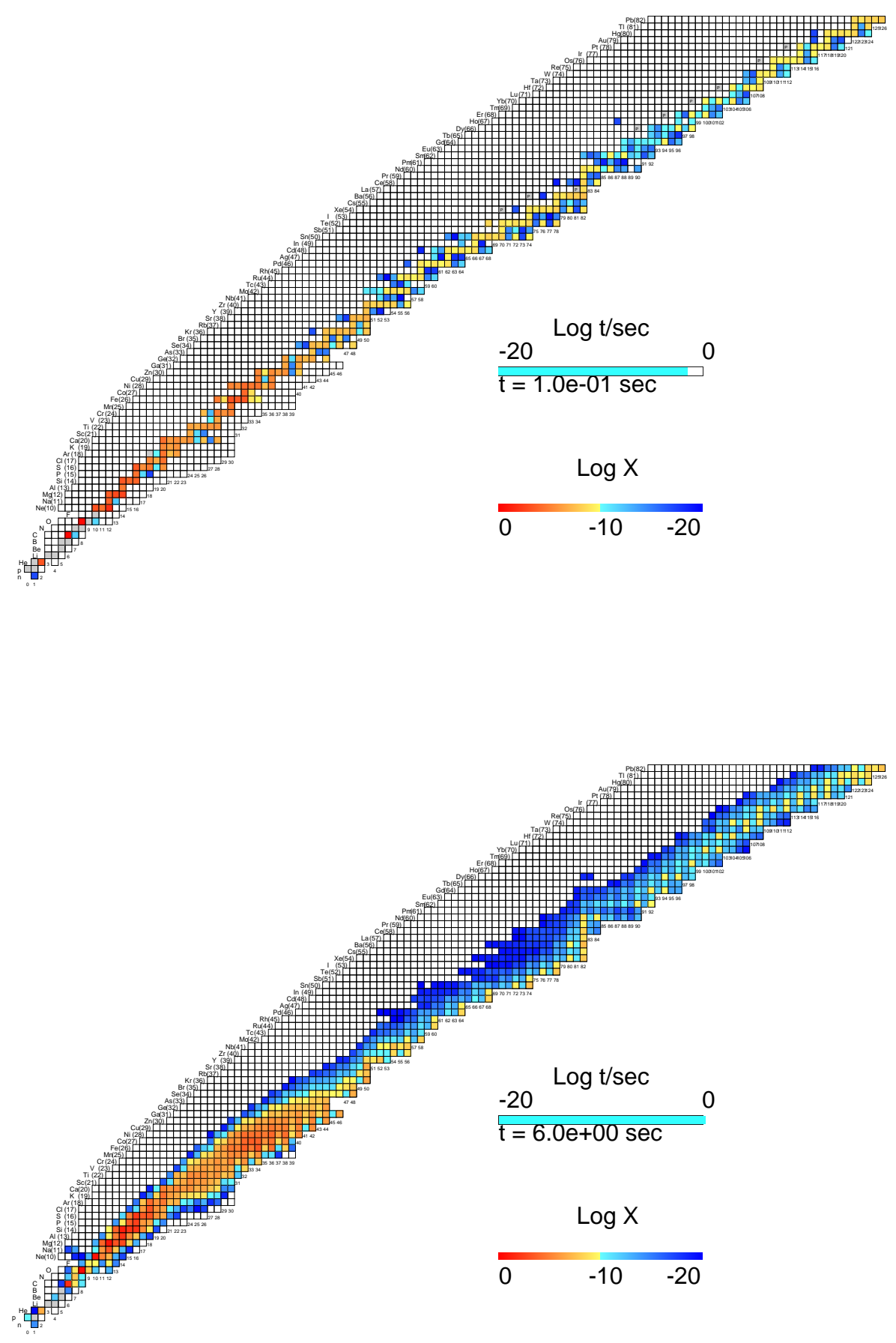

Figure 2: Abundance flow of particle where initial position is 15 degrees from the rotation axis. Upper: 1.0 sec and lower: $6.0 \mathrm{sec}$ after the core bounce. 

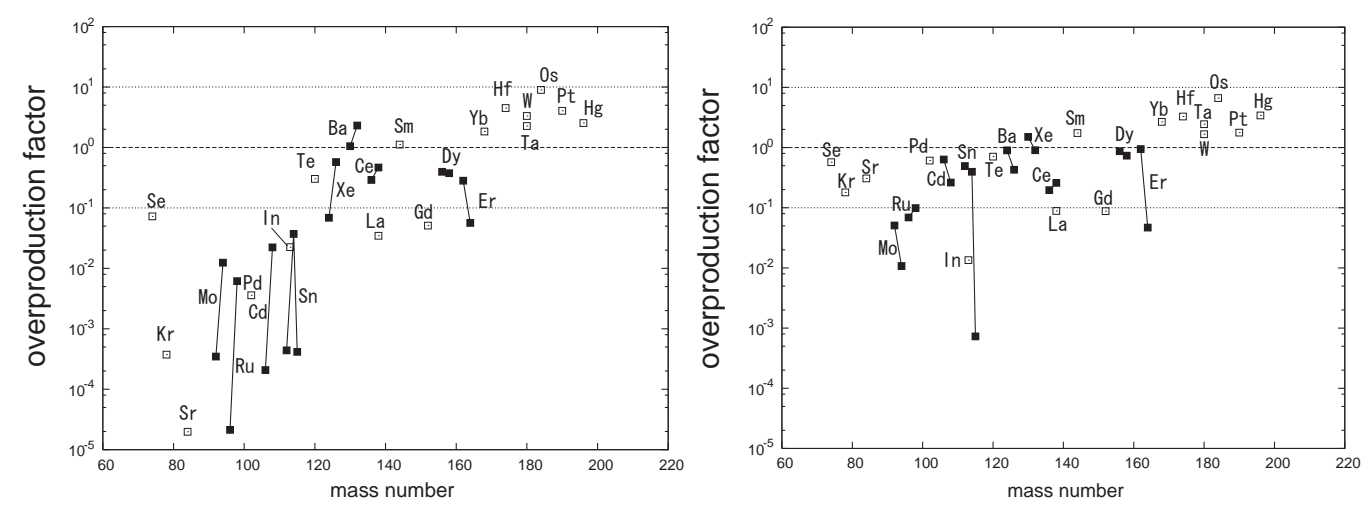

Figure 3: Overproduction factors of the $p$-elements from two jet models. Polar direction (left) and 15 degrees form the rotational axis (right).

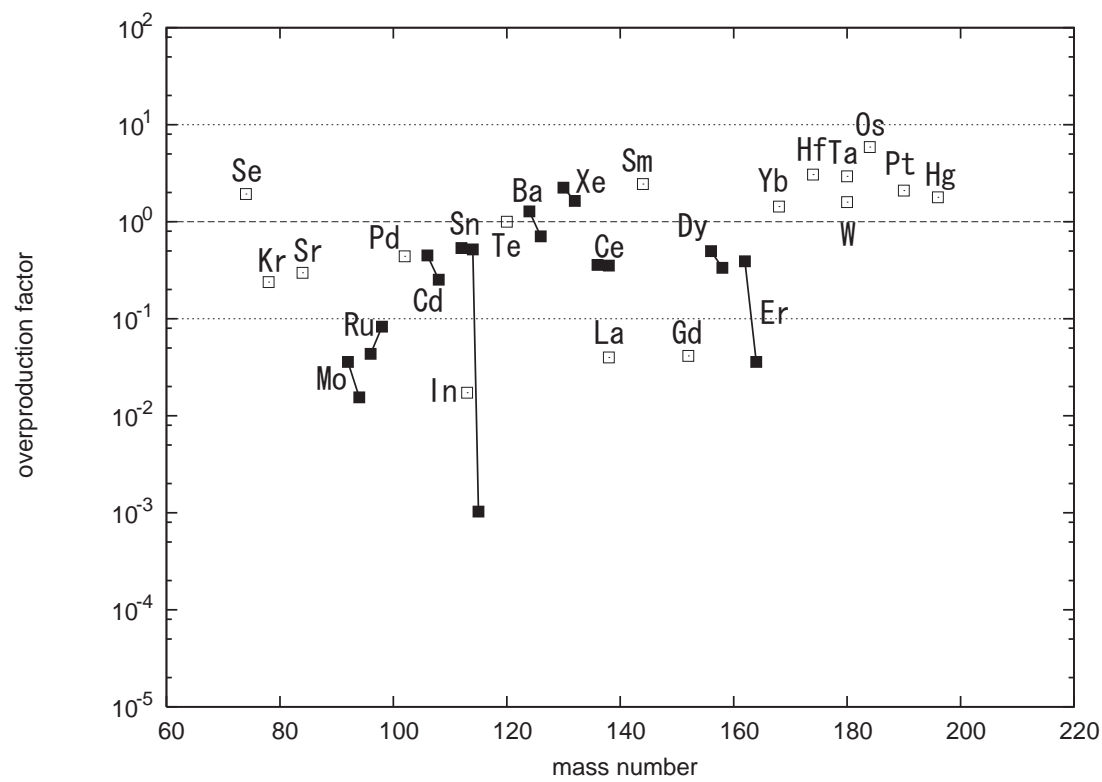

Figure 4: Overproduction factors summered over all ejected particles.

studied with use of spherical symmetric explosion models, where significant deficiencies in some $p$-elements compared to solar ones have been found. We have presented first non-spherical effects on the production of $p$-elements using the MHD explosion model. We find that the production of the $p$-elements depends on the jet direction significantly. Figure 4/shows the overproduction factor relative to the solar values. The abundances are summed over all ejected particles. We can see that deficiency of in the $p$-element production are improved well. We point out that there are variations in the heavy element nucleosynthesis such as the $r$ - and $p$-process when the MHD effects play an important role in the supernova explosion. 


\section{References}

[1] M. Rayet, M. Arnould, M. Hashimoto, N. Prantzos and K. Nomoto, The p-process in Type II supernovae, Astronomy and Astrophysics, 298, 1995

[2] S. Nishimura, K. Kotake, M. Hashimoto, S. Yamada, N. Nishimura, S. Fujimoto and K. Sato, r-Process Nucleosynthesis in Magnetohydrodynamic Jet Explosions of Core-Collapse Supernovae, The Astrophysical Journal, 642, 2006

[3] M. Hashimoto, Supernova Nucleosynthesis in Massive Stars, Progress of Theoretical Physics, 94, 663,1995

[4] K. Kotake, S. Yamada and K. Sato, Gravitational radiation from axisymmetric rotational core collapse, Physical Review D, 68, 2003

[5] T. Takiwaki, K. Kotake, S. Nagataki and K. Sato, Magneto-driven shock waves in core-collapse supernovae, The Astrophysical Journal, 616, 2004

[6] S. Fujimoto, M. Hashimoto, O. Koike, K. Arai and R. Matsuba, p-Process Nucleosynthesis inside Supernova-driven Supercritical Accretion Disks, The Astrophysical Journal, 585, 2003 